\title{
CORRELATING DEFORMATION MECHANISMS WITH MECHANICAL PROPERTIES IN FREESTANDING THIN METALLIC FILMS
}

\author{
M. A. Haque ${ }^{1}$, M. T. A. Saif ${ }^{1}$, J. S. Robach ${ }^{2}$, and I. M. Robertson ${ }^{2}$ \\ Departments of Mechanical and Industrial Engineering ${ }^{1}$ and Materials Science and \\ Engineering $^{2}$, University of Illinois at Urbana-Champaign, IL, 61801, U.S.A.
}

In this paper, we demonstrate a novel uniaxial tensile test device for use in either a scanning or a transmission electron microscope that allows direct correlation of mechanical properties with deformation and fracture processes in freestanding thin metallic films. The device, which is fabricated on a Si wafer, consists of a sensor beam to measure the applied load through its curvature, two markers to allow determination of the displacement, springs and support bars to ensure true uniaxial loading. The device is shown schematically in Figure 1. The two holes on either side are for securing it to a straining holder. The freestanding, thin metallic film has a uniform thickness and cross-section with a fine-grained microstructure. The film thickness is variable and is limited only by the electron penetration depth for the TEM case. The grain size distribution can be varied by altering the deposition conditions. Integrating sample fabrication into production of the micromachine offers a number of advantages: It ensures perfect alignment of the specimen with the loading direction, eliminating the problems with flexural stresses. The specimen is "gripped" by the micromachine through the natural adhesion of the metal with the silicon substrate, which obviates the need for a "gripping" fixture and provides a stable and firm grip. Figure 1(b) shows an SEM image of a fabricated test chip, with a $200 \mathrm{~nm}$ thick Al film.

The micrographs shown in Figure 2a show the distribution of grain sizes in a $200 \mathrm{~nm}$ thick Al film. The grains, as revealed by tilting experiments, were columnar and showed no evidence of grain boundary microcracks or pores. Dislocations were difficult to find and were located only in some of the larger grains. An example of a dislocation array extending across a grain is shown in Figure 2b. Isolated dislocations were observed but these moved rapidly and were incorporated readily in to the grain boundary. An example of isolated dislocations moving is shown in the series of images presented in Figure 3. These images were captured from individual frames from a videotape. The arrow indicates the mobile dislocation and its eventual incorporation into the grain boundary. Once in the grain boundary, the lattice dislocation was eliminated probably due to the rapid spreading kinetics in the grain boundary, removing evidence of dislocation motion.

Examination of the fracture surface in a failed specimen revealed complex dislocation arrays in many grains but only along the crack flank. There was no evidence of slip transmission between grains along the crack flank or into the interior. These results will be considered in terms of current models for improvement in mechanical properties of fine-grained systems.

Fig. 1. a) Schematic of the uniaxial test micromachine. b) SEM image of a fabricated micromachine.

Fig. 2. a) Electron micrograph showing the grain structure of a $200 \mathrm{~nm}$ thick film of Al. b) dislocation structure in one of the larger grains.

Fig.3. Dislocation motion in a fine-grained sample. 
a

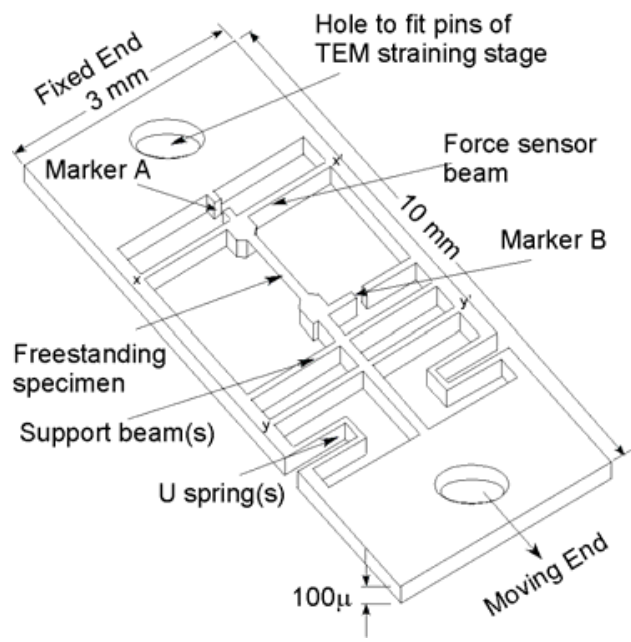

b
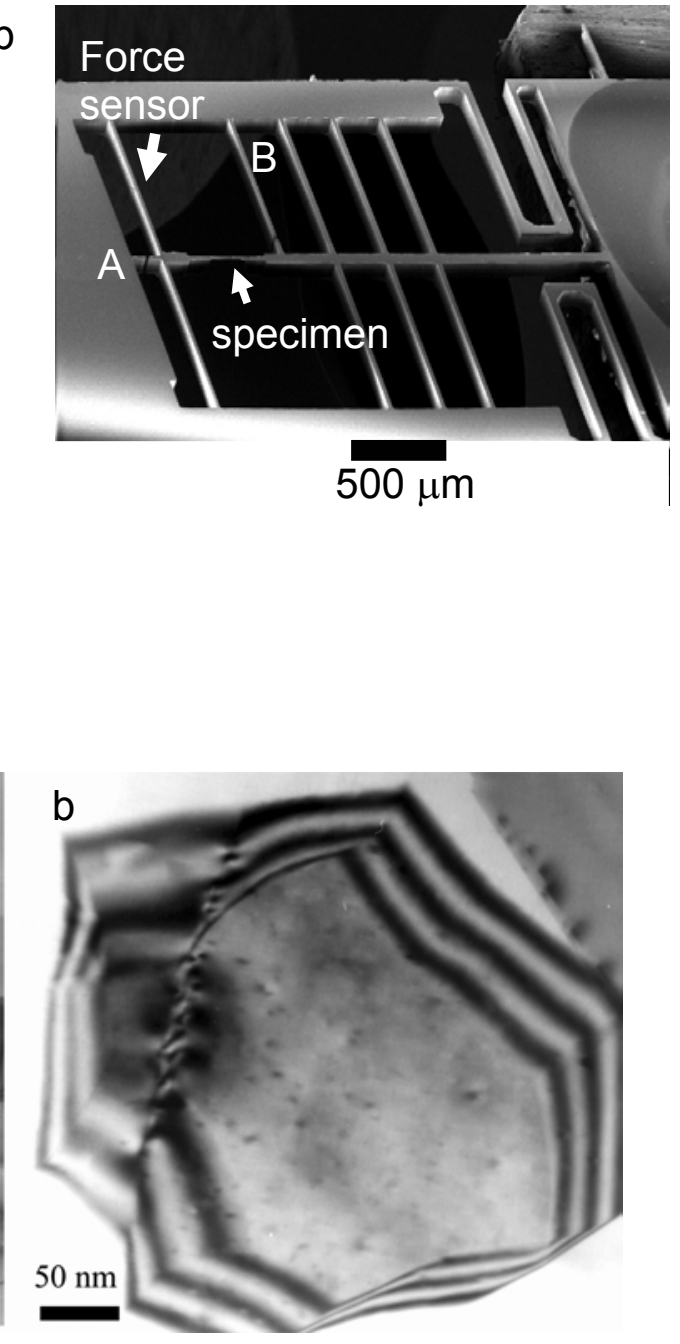

Figure 1.

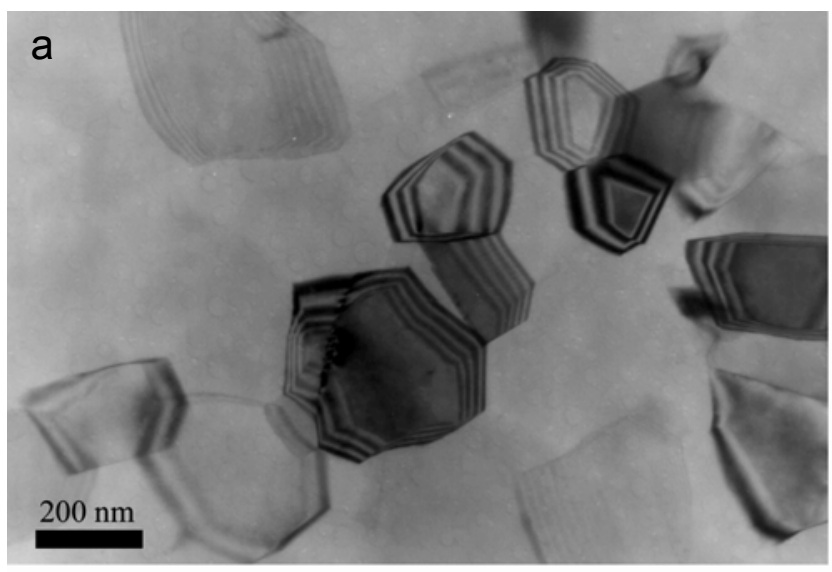

Figure 2
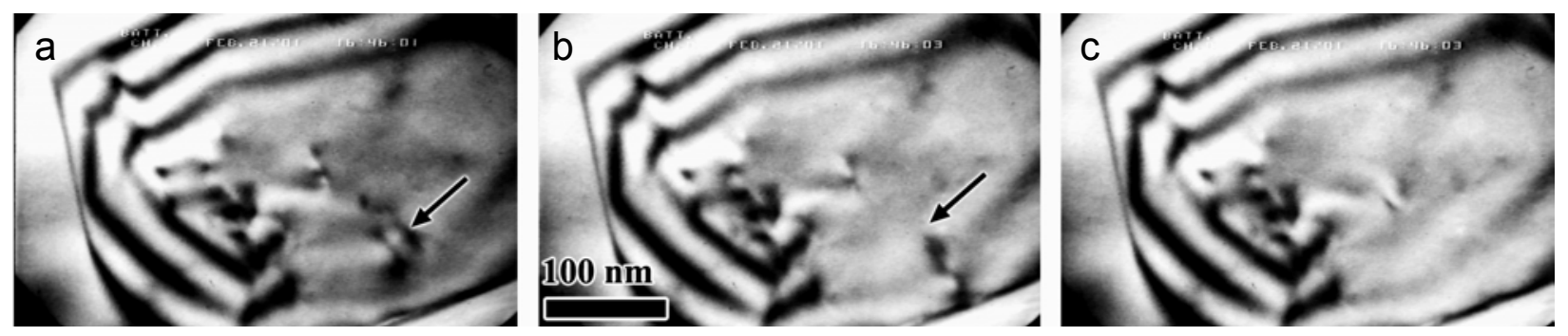

Figure 3 